By: M. Parker and Jane E. Myers

Parker, M. \& Myers, J. E. (1991). From ANWC to AMCD: Goals, Services, and Impact. Journal of Multicultural Counseling and Development, 19(1), 52-64.

*** Note: Reprinted from Journal of Multicultural Counseling and Development. The American Counseling Association. Reprinted with permission. No further reproduction authorized without written permission from the American Counseling Association: http://www.counseling.org/

\title{
Article:
}

In 1969, the American Personnel and Guidance Association (APGA, later renamed the American Association for Counseling and Development (AACE)]) established a special office for the purpose of identifying and addressing the concerns of non-White members (McFadden \& Lipscomb, 1985). After many heated discussions, one of which was about the name, the Association for Non-White Concerns in Personnel and Guidance (ANWC) became the ninth division of APGA. The goals established for ANWC in 1972 have not changed (McFadden, 1986), even though the name of the organization changed in 1985. It is timely, then, to review the historical development of what is now the Association for Multicultural Counseling and Development (AMCD) and the impact of the name change. In so doing, it is important also to examine the goals and services of this organization.

\section{HISTORICAL DEVELOPMENT OF ANWC/AMCD}

The civil rights movement of the 1960s was an important stimulus to the development of ANWC. During that era, African American persons were becoming concerned with their civil rights, developing pride in their ethnicity, and demanding equality of treatment in every aspect of human endeavor including their professional involvements. In this regard, African American members of APGA insisted that they be included in the core structure of the organization, using ANWC as their vehicle for inclusion.

The fledgling organization struggled to define its focus as being multiethnic or primarily African American. Some felt that ANWC should focus on minorities in general, while other members believed that the new organization should address the specific needs of African Americans, the largest minority in APGA (McFadden \& Lipscomb, 1985). The controversy has not been fully resolved, as exemplified in the continuing question should AMCD be AACD's division for African Americans, or should it be the division for any association member dealing with multicultural, multiethnic, or cross-cultural issues and concerns?

Since 1972, many assumptions have been made concerning ANWC/AMCD's goals, objectives, roles, and functions among APGA/AACD divisions. Many APGA/AACD members perceive ANWC/AMCD as the division for African Americans' problems and concerns rather than for a broader range of individuals and groups. Some view members of this division as the militant group from the 1960s, still mainly concerned with civil rights, prejudices, and racial injustices.

A pervasive concern is that negative societal stereotypes of African Americans have affected APGA/AACD members' perceptions of ANWC/AMCD. For example, ANWC/AMCD is sometimes described as the division that has the best dance or social hour at the annual national convention. The name non-White has contributed to the stereotypical perceptions, leaving ANWC/AMCD's goals, services, and functions largely misunderstood both within the division and within AACD.

White members having distinctive cultural or ethnic status (e.g.. Cuban Americans) have been confused or even frustrated by the name non-White, not knowing if this is an appropriate division with which they should affiliate. Many such individuals have not joined, supported, or understood ANWC/AMCD's mission. 
Unresolved perceptions and disagreements concerning ANWC/AMCD's mission, role, and function eventually led to the change of its name, in 1985, to AMCD.

\section{WHAT'S IN A NAME?}

The APGA changed its name to AACD shortly before the AMCD name change. This change occurred at a critical time, when the counseling needs of culturally diverse groups were becoming increasingly apparent (Parker, 1988). The growth of other minority groups (e.g.. Hispanic Americans and Asian Americans) stimulated increasing concern for a broadening of the definition of possible members of AMCD, to include a multiethnic and not just an African American focus. It would be interesting to know at this point in AMCD's history, what, if any, impact the name change has had on the current memberships and perceptions of other AACD members of this division. Opponents of the name change believed it would result in a decline in African American membership, weakening of African American identity, and decline in the overall effectiveness of the division in achieving its goals. Others believed that the name change would stimulate a more multiethnic membership, including more White Americans.

Five years since the name change, many African American AMCD members believe that the organization they initiated to meet their needs has been eliminated and masked under the rubric of multiculturalism. Others support the name change and credit it with the growth of the AMCD membership from 1,406 in 1984 to 2,610 in fall, 1989. These persons believe the quality of services has improved, and the association is better able to meet the challenges of serving multicultural populations in the next decade.

The name change was made, as mentioned, without a change of goals or services. In view of the many societal changes that have occurred since the founding of this division in 1972, and the resulting challenges to the counseling and human development professions, a reexamination of AMCD's goals is timely and necessary. Because goals dictate services, a review of services provided to members is an integral part of this examination. It is important for AMCD's leaders to be aware of members' perceptions of goals and services and also the desires of members for new or improved services. These data would provide direction for organizational planning for AMCD leadership.

\section{METHODOLOGY}

After careful review of the history of ANWC/AMCD and discussion with AMCD members and leaders, a survey was developed to assess AMCD's goals, objectives, and services and the impact of the name change. A pilot test of the instrument was conducted and suggestions for revisions were provided. Participants in the pilot test included members of several AACD divisions, including AMCD. Specialists in multicultural issues, association issues, and assessment were among those included in the initial test group. Results and feedback from the pilot test led to the appropriate revisions.

The survey used in this study included five sections: (a) identifying information, (b) purposes of AMCD and impressions of AMCD's progress toward its purposes, (c) general impact of the name change, (d) impact of the name change on AMCD's journal and newsletter, and (e) AMCD's services. Opportunities for comments from respondents were provided in each section.

The survey was mailed to a random sample of 500 AMCD members and 500 AACD members $(\mathrm{N}=1,000)$. A self-addressed, stamped envelope was included to assist prompt return of completed surveys.

\section{RESULTS}

A total of 247 surveys were returned, for a response rate of $24.7 \%$. Of these, $54(22 \%)$ were members of AMCD and $193(78 \%)$ were members of other divisions. The largest numbers of responses from AACD members were from the largest divisions. These included 22 from the American School Counselor Association (9\%), 19 from the American College Personnel Association (8\%), 16 from the American Mental Health Counselors Association (7\%), and 11 (4.5\%) each from the Association for Counselor Education and Supervision and the 
Association for Specialists in Group Work. Other divisions had 10 or fewer responses. At least two surveys were received from each division.

Part I, demographic information, is shown in Table 1. The totals for the various categories are not the same due to missing responses to some questions. The most common employment position was that of counselor $(\mathrm{N}=49$, $20 \%)$, working in a college or university $(\mathrm{N}=33,13 \%), \mathrm{K}-12$ setting $(\mathrm{N}=24,10 \%)$, or community agency $(\mathrm{N}=37,15 \%)$. Almost all respondents $(96 \%)$ had a bachelor's degree or higher, and $92(89 \%)$ had a master's degree or higher. Forty respondents $(40 \%)$ were men and $60(60 \%)$ were women. Fifty-nine $(60 \%)$ respondents were Caucasian and 40 (40\%) reflected other ethnic backgrounds.

In Part II of the survey, a list of the seven official purposes of AMCD was provided. Respondents were asked to (a) indicate their extent of agreement with whether the statement should be a purpose of AMCD, (b) rank order the purposes from most to least important, and (c) rate the extent to which AMCD is currently addressing its purposes. Responses to these questions are provided in Table 2 (see p. 57). Mean scores are based on a scale of 1 to 5 , with 1 being strongly disagree and 5 being strongly agree.

The mean score for six of the seven purposes, according to whether each should be a purpose, ranged between 4.28 and 4.65. Only one. Purpose 4 (see Table 2), obtained a mean score of less than 4.0.

The ranking of purposes did not correspond exactly to the mean scores. Here, Purposes 5 and 1 were ranked highest, while 6 and 7 were ranked lowest. Ranks were determined by computing the overall frequency of rank order assigned to each purpose.

Part III of the survey assessed the impact of the AMCD name change. Responses to this part are shown in Table 3 (see p. 58). Mean scores reflect a scale from 1 to 5, with 1 being strongly disagree and 5 being strongly agree. Many respondents (12\%) indicated in their comments that they did not have enough familiarity with AMCD to respond to this section. Some of these respondents were new AACD members who had limited knowledge of AMCD as one of the divisions of AACD. Others reported that they did not know that the name change was an issue, because they never heard it discussed or debated in meetings.

Another small group stated they were simply unaware of the name change.

Of the 12 mean scores reported in this table, 10 ranged between 3.07 and 3.95. These scores indicated some, but not strong, agreement with the impact statement. Two of the mean scores (Impacts $5 \mathrm{~d}$ and 8, see Table 3) were below 3.0, indicating some disagreement. In other words, respondents did not feel that the name change caused a decline in African American members or weakened the division as a focal point for the identity of African American AACD members.

In Item 9 of Part III, respondents gave their opinions on what they believe has been the major impact of the name change. Ninety-six persons (39\%) did not respond. The overwhelming majority of the remaining respondents believed the name change has had a positive impact on the organization and its members, while a much smaller number of respondents believed the name change has had a negative effect. Many of the respondents made positive comments based on their perceptions of the name change. The most representative positive comments are listed as follows:

1. There has been a greater awareness and sensitivity to all cultures.

2. The name change represents a more contemporary approach to cross-cultural issues.

3. The new name makes AMCD more accessible and welcoming to majority group members of AACD. 
4. With the name change there has been a move from narrow thinking to embracing the more global picture.

5. The new name presents an image that is more comfortable, less threatening, less hostile, less biased, less prejudiced, more inviting, and more relevant to a pluralistic society.

6. The name change has projected a more growth-oriented focus.

7. $\quad$ The name change has begun to decrease the negative connotation(s) of "non-White."

8. There has been a clearer understanding of who is eligible for AMCD membership.

9. There has been more cooperation between the various minority groups.

10. The name change represents a positive concern rather than a discriminatory title. "How would we as Blacks feel if we were part of an organization with a group with 'non-Black concerns' in the title? We would be the first to yell 'discrimination."'

11. The name change has contributed to an increase in membership and an improved awareness of AMCD as an integral part of AACD.

Those respondents who were not supportive of the name change made comments such as the following:

1. The name change has defeated the original purpose, which was the self-improvement of African American AACD members.

2. There has been a decrease in African American identification with the AMCD organization.

3. The AMCD's focus on refugee status and the change of the U.S. population place African American representation in the back seat.

4. The name change has caused dissension among members, supporters, and interested parties of AMCD.

5. The name change to AMCD has caused confusion with AMECD (the Association for Measurement and Evaluation in Counseling and Development), causing people to select the wrong convention programs and so forth.

Part IV of the survey assessed the impact of the name change on the AMCD journal (the Journal of Multicultural Counseling and Development) and newsletter (AMCD Newsletter). A summary of responses to this part of the survey, again using a scale of 1 to 5 , is shown in Table 4.

Mean scores for questions about the journal ranked from 3.37 to 3.91, while those concerning the newsletter ranked from 3.24 to 3.62. About one-third (37\%) of respondents noted that they read the newsletter regularly. Part V solicited opinions regarding the services AMCD provides. Responses to this part are provided in Table 5. As can be seen from this table, more than half of the respondents were unable to comment on AMCD's services. Most respondents who evaluated the services thought that they were satisfactory. In general, more persons thought that each service needs improvement than that the service was exemplary.

\section{DISCUSSION}

Each part of the survey merits specific comments, as indicated in the following discussion. 


\section{Part I: Demographic Information}

The response rate of $24 \%$ appears adequate for interpretive purposes. The sample is generally representative of AACD membership. The low response rate from AMCD members $(22 \%)$ is noteworthy, as AMCD represented $50 \%$ of the original sample. This low response rate may reflect or may be an indication of general apathy in the division. Additionally, it might be that some AMCD members are not fully informed of the services provided to enable them to respond (see following discussion of Table 5). An alternative explanation could be that AMCD members, like many other AACD members, are so overcommitted that filling out a 20-minute survey is perceived as a lowest priority task.

\section{Part II: Perceptions of AMCD's Purposes}

The data revealed that all seven of the original division purposes were perceived as valuable by the respondents. The two most important (or highest ranked) were Purposes 1 and 5, whereas the lowest was 6. The pattern here seems to be that the values of securing equality of treatment and promoting a greater understanding of ethnic minority groups are seen as more important than specific means or procedures to accomplish the values (such as publishing a journal and other scientific materials). This is consistent with the tendency we have noticed for professionals to subscribe to principles (such as believing that counselors should be trained in ethnic concerns) while they are less likely to practice these principles (such as participating in continuing education workshops to update their own skills in multicultural counseling).

\section{Part III: Impact of Name Change}

In general, respondents indicated that the division name change had a positive impact including being more sensitive toward other cultures, being more contemporary in its approach to cross-cultural issues, being more open to majority members, having a greater global vision, having a more growth-oriented focus, having greater cooperation between minority groups, having increased membership, and having clarified goals.

In addition, it seems that the name change has not had the negative impact on African American members that had been anticipated (i.e., the majority of respondents did not feel the name change caused a decline in African American membership or that it had weakened the division as a focal point for the identity of African American AACD members). The comments volunteered by respondents regarding their perceptions of the name change's impact were overwhelmingly positive as well. A small percentage of the respondents, however, did have negative perceptions of the impact of the name change for African American members of AACD.

We conjecture that those comments reflecting a negative opinion of the change were made by the same people who were apprehensive during initial deliberations about the name change. This would seem logical from a historical context, because these respondents may be the same people who championed the need for representation of minority (non-White) concerns in the early 1970s and were instrumental in the formation of the division. The efforts of these dedicated pioneers in establishing the division might (understandably) make it difficult for them to accept expanding the focus and membership of the division to include minority groups other than African Americans.

\section{Part IV: Impact of Name Change on AMCD's Journal and Newsletter}

Respondents indicated that the journal and newsletter were generally perceived as having improved since the name change of the association. If the journal and newsletter have improved, there is insufficient evidence to explain this observation. The respondents were even stronger in agreeing that the journal and newsletter were relevant to the needs of both the profession as a whole and themselves as individuals.

\section{Part V: Opinions Regarding the Services AMCD Provides}

The respondents generally endorsed the services AMCD provides, with more respondents finding services satisfactory or exceptional than needing improvement. Leadership was the most positively perceived service (by a 4:1 ratio), with the national conference and publications strongly endorsed as well (by a 3:1 ratio). Legislative advocacy was perceived as marginally satisfactory. 
Although Table 5 is in general a positive "report card" for the services offered by the division, there is a clear perception that services need improvement. Perhaps most important of all is the large percentage of respondents who indicated they were unaware that the division is providing these services. These data suggest that the division leadership needs to address how to better inform members of the services and activities the division is providing. The data also suggest that the membership must take more responsibility to remain informed and involved if AMCD is to continue to be a viable organization for representing multicultural concerns.

\section{CONCLUSION}

Although all of AMCD's purposes were viewed as valuable, those purposes concerned with securing equality of treatment and promoting greater understanding of ethnic minorities were the most important ones. Since the name change there has been a greater attempt to understand, to meet the needs of. to provide services for, to be more accepting of, and to promote cooperation between ethnic minority groups. The name change has also projected a more positive image of AMCD that many respondents believe is a contributing factor toward the increase in AMCD's members.

Change rarely occurs without some degree of doubt, fear, and apprehension. Some respondents viewed the name change with skepticism, believing that the original purposes of AMCD as being a focal point for African American identity issues and concerns may be overshadowed under the label of multiculturalism. Those who are most skeptical are probably the early founders of the ANWC organization who, because: of their many years of dedicated service, find it difficult to accept expanding the focus to include groups other than African Americans.

The AMCD's leadership, national conference, and publications were perceived by respondents as satisfactory even though services in general need improvement.

An important finding was that respondents were unaware of many services and activities offered by AMCD. This finding may well offer AMCD's leadership the major challenge of the decade, which is informing members better, stimulating member involvement, and continuing progress toward division goals.

Overall, the: respondents felt that the impact of the name change is positive and AMCD is taking steps in the right direction.

Authors' Note. Special thanks are extended to Drs. Barbara Brown Robinson, NCC, Director, Association Relations of AACD, Alexandria, Virginia, and Janice Jordan, Assistant Director, Counseling and Student Development, University of Delaware, Newark, for their assistance in reviewing and refining the survey and methodology used in this study.

\section{TABLE 1 Demographic Information Provided by Respondents}

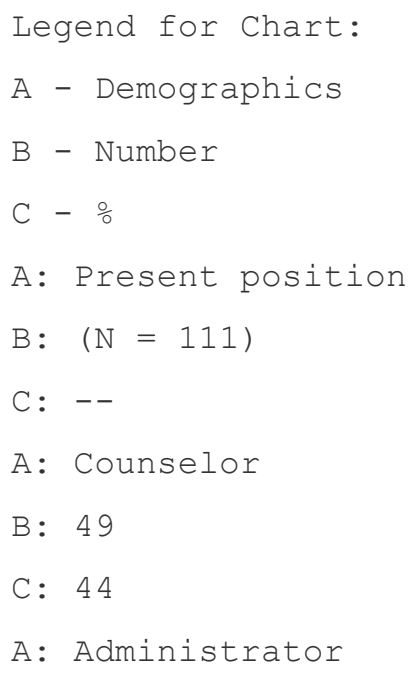


B: 12

C: 11

A: Counselor education supervisor

B: 14

C: 13

A: Student

B : 9

$\mathrm{C}: 8$

A: Paraprofessional

$B: 8$

C: 7

A: Other

B : 19

C: 17

A: ork setting

B : $\quad(\mathrm{N}=126)$

C: --

A: College or university

B: 33

C: 26

A: $\mathrm{K}-12$

B: 24

C: 19

A: Community college

B : 5

C: 4

A: Community agency

B: 37

C: 29

A: Institutional setting

B: 8

C: 6

A: Business or industry

B: 2

C: 1.5

A: Other

B: 17

C: 13

A: Education

B : $\quad(N=104)$ 
C: - -

A: Doctorate

B: 25

C: 24

A: Educational, specialist

B: 8

C: 8

A: Master's

B: 59

C: 57

A: Bachelor's

B: 8

C: 8

A: Other

B: 4

C: 4

A: $\operatorname{Sex}$

B: $(N=100)$

C: - -

A: Male

B: 40

C: 40

A: Female

B: 60

C: 60

A: Annual income

B: $\quad(\mathrm{N}=111)$

C: - -

$\mathrm{A}:<14,999$

B: 13

C: 12

A: $15,000-24,999$

B: 21

C: 19

A: $25,000-34,999$

B: 39

C: 35

A: $\quad 35,000-49,999$

B: 24

C: 22 


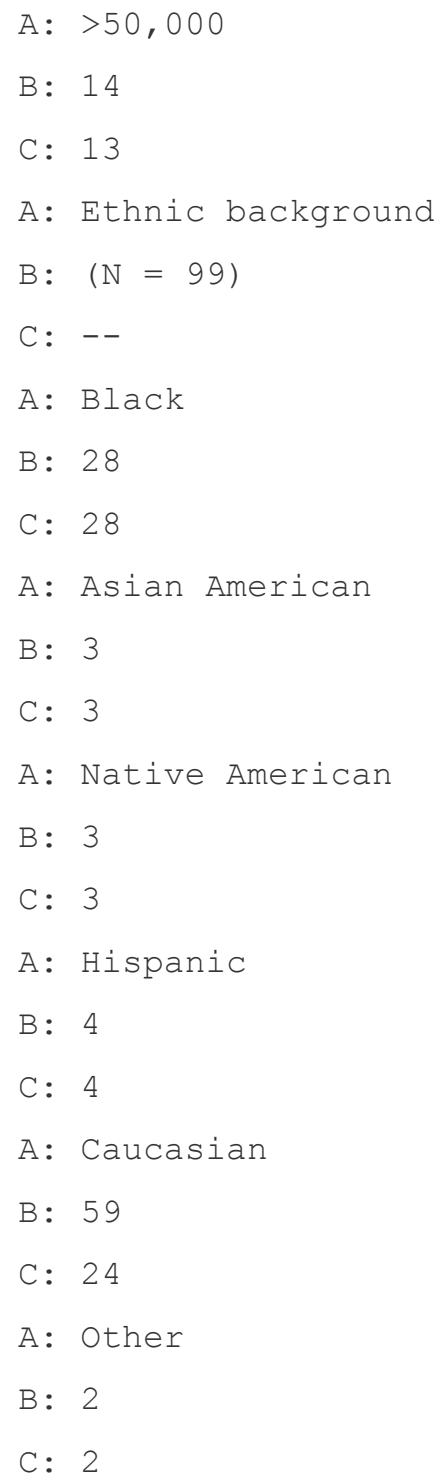

\section{TABLE 2: Reactions to AMCD Purposes}

Legend for Chart:

A - Purpose

B - Should be a purpose: M

C - Should be a purpose: SD

D - Rank order

E - Progress toward purpose: M

F - Progress toward purpose: SD

A: 1. To promote a greater understanding of ethnic minority groups among members of the counseling and guidance professions

B: 4.74

C: .61

$\mathrm{D}: 2$

$E: 3.76$

$\mathrm{F}: .82$

A: 2. To improve the standards of counseling and guidance given to 
ethnic minority groups

B: 4.65

C: .70

D: 4

$\mathrm{E}: 3.58$

$\mathrm{F}: .85$

A: 3. To identify and work to eliminate conditions that create barriers to the individual development of ethnic minority groups

B: 4.49

$C: .79$

$\mathrm{D}: 5$

$\mathrm{E}: 3.48$

$\mathrm{F}: \quad .82$

A: 4. To develop, implement, and/or foster interest in charitable, scientific, and educational programs designed to further the interests of ethnic minority groups

B: 3.96

C: .79

$\mathrm{D}: 3$

$E: 3.33$

F: .72

A: 5. To secure equality of treatment, advancement, qualifications, and status of minorities in counseling and development work

B: 4.45

C: .84

$\mathrm{D}: 1$

$E: 3.38$

$\mathrm{F}: .83$

A: 6. To publish a journal and other scientific, educational, and professional materials with the purpose of raising the standards of all who work in counseling and guidance

B: 4.28

C: 91

D: 7

$E: 3.90$

F : .91

A: 7. To facilitate a broader awareness of multicultural counseling among the helping professions

B: 4.59 
F: 84

\section{TABLE 3: Impact of Name Change From ANWC to AMCD}

Legend for Chart:

A - Impact

B - M

C $-S D$

A: 1. Helped other divisions of AACD better understand AMCD's roles and functions

B: 3.73

C: 0.89

$A: 2$. Helped AMCD members better understand the roles and functions of the division

B: 3.60

C: 0.89

A: 3. Broadened the scope of AMCD's goals and purposes

B: 3.95

C: 0.88

A: 4. Clarified the scope of AMCD's goals and purposes

B: 3.79

C: 0.90

A: 5. Affected the ethnic composition of AMCD membership

B: 3.44

C: 0.88

A: a. Resulted in more White American members of AMCD

B: 3.24

C: 0.82

A: b. Resulted in a wider variety of ethnic minority members of AMCD

B: 3.42

C: 0.80

A: C. Resulted in more Black American members of AMCD

B: 3.07

$C: 0.67$

A: d. Caused a decline in Black American members of AMCD

B: 2.78

C: 0.65

A: 6. Broadened the ethnic minority representation among AMCD's leadership 
B: 3.27

C: 0.73

A: 7. Strengthened the AMCD journal

B: 3.54

C: 0.79

A: 8. Weakened the division as a focal point for the identity of Black AACD members

B: 2.87

C: 0.88

TABLE 4 Impact of Name Change on AMCD Journal and Newsletter

Legend for Chart:

A - Impact

$\mathrm{B}-\mathrm{M}$

$C-S D$

Journal

A: 1. The AMCD journal has improved since the AMCD name change.

B: 3.37

$\mathrm{C}: 0.74$

A: 2 The AMCD journal is relevant to the needs of the counseling profession.

B: 3.91

C: 0.84

A: 3. The AMCD journal is relevant to your needs.

B: 3.76

C: 0.90

Newsletter

A: 1. The AMCD newsletter has improved since the name change.

B: 3.24

$C: 0.65$

A: 2. The AMCD newsletter is relevant to the needs of the counseling profession.

B: 3.62

$\mathrm{C}: 0.79$

A: 3. The AMCD newsletter is relevant to your needs.

B: 3.45

$\mathrm{C}: 0.81$

TABLE 5 Opinions Regarding the Services AMCD Provides

Legend for Chart:

A - Service

B - Does not know 


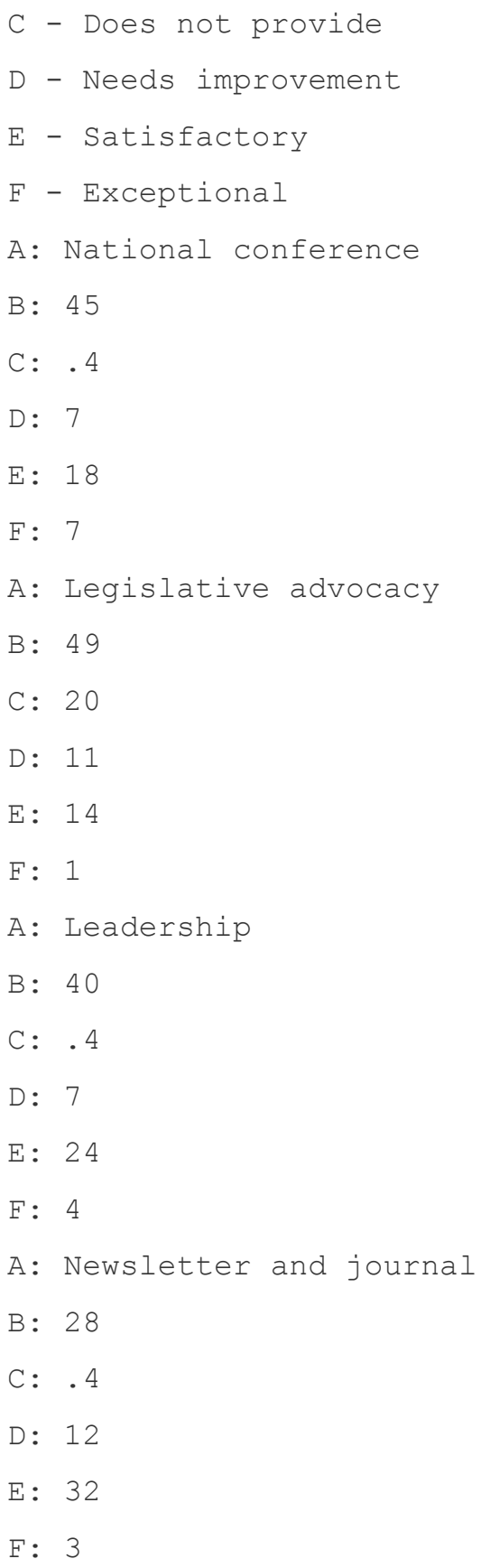

\section{REFERENCES}

McFadden, J. (1986). Policies and procedures manual. Alexandria. VA: Association for Multicultural Counseling and Development.

McFadden, J., \& Lipscomb, W. D. (1985). History of the Association for Non-White Concerns in Personnel and Guidance. Journal of Counseling and Development, 63, 444-447.

Parker, W. M. (1988). Consciousness-raising: A primer for multicultural counseling. Springfield. IL: Thomas. 